\title{
Targeting regressions: Do readers pay attention to the left?
}

\author{
Jens K. Apel • John M. Henderson • Fernanda Ferreira
}

Published online: 12 July 2012

(C) Psychonomic Society, Inc. 2012

\begin{abstract}
The perceptual span during normal reading extends approximately 14 to 15 characters to the right and three to four characters to the left of a current fixation. In the present study, we investigated whether the perceptual span extends farther than three to four characters to the left immediately before readers execute a regression. We used a display-change paradigm in which we masked words beyond the three-to-four-character range to the left of a fixation. We hypothesized that if reading behavior was affected by this manipulation before regressions but not before progressions, we would have evidence that the perceptual span extends farther left before leftward eye movements. We observed significantly shorter regressive saccades and longer fixation and gaze durations in the masked condition when a regression was executed. Forward saccades were entirely unaffected by the manipulations. We concluded that the perceptual span during reading changes, depending on the direction of a following saccade.
\end{abstract}

Keywords Eye movements and reading · Attention · Regressions

During reading, people perceive information within their perceptual span (McConkie \& Rayner, 1976; Rayner, Well, $\&$ Pollatsek, 1980). The perceptual span defines the area in which effective visual processing during reading is possible. When reading English sentences, this area is asymmetric to the right, spanning three to four characters to the left and 14

J. K. Apel ( $\square)$

General and Biological Psychology, University of Wuppertal,

42119 Wuppertal, Germany

e-mail: japel@uni-wuppertal.de

J. M. Henderson · F. Ferreira

Department of Psychology, University of South Carolina,

Columbia, SC, USA to 15 characters to the right of a given fixation (McConkie \& Rayner, 1975, 1976; Rayner \& Pollatsek, 1989; Underwood $\&$ McConkie, 1985). Only very little information is acquired from outside the perceptual span (Rayner \& Bertera, 1979; Rayner, McConkie, \& Ehrlich, 1978).The reason for this asymmetry is that, in reading, before the eyes move to a new location, attention moves first (Henderson, Pollatsek, \& Rayner, 1989). Morrison (1984) compared this process with the analogy of a rubber band: Attention moves first and the eyes are pulled behind, as if they were attached to it with a rubber band. The perceptual span allows text to be preprocessed, and the resulting information is used to plan a saccade to the next location (Rayner, 1975).

In such languages as Hebrew, in which the normal reading direction is from right to left, the perceptual span extends farther to the left of fixation than to the right (Pollatsek, Bolozky, Well, \& Rayner, 1981). Likewise, when readers of English are required to read English text from right to left, the perceptual span also extends farther to the left (Inhoff, Pollatsek, Posner, \& Rayner, 1989).

In the present study, we were interested in whether the perceptual span in reading is always directed toward the general reading direction of a language, or whether it changes within a language depending on the intended direction of the following saccade. In English, a saccade is directed to the right in progressions and to the left in regressions. Progressions are the predominant eye movements in reading, but $10 \%-15 \%$ of all eye movements are regressions (Rayner \& Pollatsek, 1989; Vitu \& McConkie, 2000). Readers regress for a number of reasons, such as in response to low-level visuomotor or word identification processes, or due to higher-level syntactic and semantic processes (Rayner, 1998; Vitu, 2005). Regressions due to visuomotor and word identification processes are usually very short and consist mostly of interword regressions or regressions that land on the word immediately to the left of the launch site. 
Regressions due to higher-level processing problems are usually longer, and the length of regressive saccades can cover multiple words (Frazier \& Rayner, 1982; Meseguer, Carreiras, \& Clifton, 2002).

In the present study, we were interested only in regressions caused by higher-level processes. We investigated whether the perceptual span extends farther than three to four characters to the left when readers are about to execute a regressive eye movement caused by syntactic confusion.

Most previous studies investigating the perceptual span have used the moving-window technique (McConkie \& Rayner, 1975): While a participant reads through a sentence, letters to the left and right of the current fixation are replaced by Xs or by different letters. By varying the number of unchanged letters around the point of fixation, it is possible to determine how many letters to the right and left are processed during a single fixation. We used a similar paradigm for the present study, but only letters to the left of a current fixation and outside of the established perceptual span of four letters to the left were replaced. If the perceptual span extends more than four letters to the left when a regression is planned and executed, our manipulation should change reading behavior for regressive but not for forward eye movements.

\section{Method \\ Participants}

A group of 32 participants from the University of Edinburgh took part in this experiment. The average age of the participants was 19.9 years (range $18-26$ ). All of the participants were native speakers of English. They had normal or corrected-to-normal vision and were each paid $£ 6$.

\section{Material}

A set of 40 garden path sentences from two previous studies (Frazier \& Rayner, 1982; Pickering \& Traxler, 1998) were used in this experiment (see Example 1 below and the Appendix). Garden path sentences contain a local syntactic ambiguity, and the incorrect interpretation of this ambiguity is initially the preferred one. Therefore, such sentences have to be reanalyzed when the disambiguating breakdown region is read. Regressive eye movements usually accompany this reanalysis (Frazier \& Rayner, 1982). The sentences used in this study were selected because they induced a high number of regressive eye movements from the breakdown region (Frazier \& Rayner, 1982; Pickering \& Traxler, 1998).

(1) While Mary was mending the clock in the dark hall frightened her with its chime.
The original sentences were slightly changed to ensure that the positions of the ambiguous verb (here, mending) and the breakdown region (frightened) were the same in every sentence. The ambiguous verb was always the fourth word and was followed by a two-word noun phrase (e.g., the clock). The breakdown region was kept constant at the eleventh word. Thus, four words were always interposed between the noun phrase and the breakdown region.

For each of the two words positioned to the left of the disambiguating region, a second, masked version was created, in which each character was replaced by a different character that was visually similar to the original character. Descending letters were replaced with descending letters (e.g., g $\rightarrow \mathrm{q}, \mathrm{p} \rightarrow \mathrm{g}$ ), ascending letters were replaced with ascending letters (e.g., $\mathrm{t} \rightarrow \mathrm{l}, \mathrm{k} \rightarrow \mathrm{h}$ ), and baseline letters were replaced with baseline letters (e.g., $\mathrm{a} \rightarrow \mathrm{n}, \mathrm{c} \rightarrow \mathrm{s}$ ). Consonants as well as vowels were replaced by consonants, resulting in masked words that consisted only of consonants. Thus, the altered strings were always nonwords.

The masked versions of the two words to the left of the disambiguating region appeared when participants fixated the disambiguating region on at least the fourth character. As mentioned above, the perceptual span in forward reading extends four characters to the left of a given fixation. Thus, the masking manipulation should have no effect on forward reading. If the reading span extends farther than four characters to the left before a regression is launched, differences in reading behavior should be observed.

The disambiguating word at the breakdown region was at least nine characters long. This ensured that readers would fixate the disambiguating word on at least the fourth character. The preferred viewing position on a word is just to the left of the word's center (Rayner, 1979), making the fourth or fifth character of a nine-character-long word a likely fixation location.

The sentences were presented in 14-point Courier New font and always fitted on a single line. Forty non-gardenpath sentences were included as fillers. The fillers consisted of simple and complex sentences and did not contain any syntactic ambiguities. These fillers were not masked.

\section{Apparatus}

An SR EyeLink1000 eyetracker with a sampling rate of $1000 \mathrm{~Hz}$ and a spatial resolution of less than $0.25 \mathrm{deg}$ was used. The tracker was tower mounted, and the eye movements of the right eye were tested. The stimuli were presented on a 19-in. CRT monitor running at $140 \mathrm{~Hz}$. The resolution of the screen was set to $1,024 \times 768$ pixels. The University of Massachusetts Amherst (UMass) software for the SR EyeLink II and EyeLink 1000 was used to run the experiment, and the analysis was done using DataViewer, developed by SR Research Ltd. and MATLAB. 


\section{Procedure}

The participants were seated in a soundproofed chamber, with the monitor located at a distance of $90 \mathrm{~cm}$. On the basis of this distance and of the size of the font, approximately 3.3 characters per degree of visual angle were displayed.

After a participant had read the instructions, the eyetracker was manually calibrated using a nine-point fixation stimulus. The EyeLink software validated the calibration. If the validation was poor, it was repeated. The calibration procedure was repeated approximately three times during the experiment. Between items, participants had to fixate a point in the middle of the screen, allowing the experimenter to perform a drift correction if necessary. Furthermore, a small rectangle was shown at the upper left side of the monitor. This rectangle was at the position of the first word of the following sentence. In order to trigger the next sentence, participants had to fixate this rectangle. Thus, when the sentence appeared on the screen, the participants' eyes were already fixating its beginning.

Words were masked when participants fixated the breakdown region on at least the fourth character. Only the first (left1) and second (left2) words to the left of the breakdown region were masked. Example 2 illustrates the sentences in the four conditions. Either no word to the left of the breakdown region was changed [left1(no mask) \& left2(no mask): Sentence 2a], the first word to the left was changed [left1 (mask): Sentence 2b], the second word to the left was changed [left2(mask): Sentence 2c], or both the first and second words to the left were masked [left1(mask) \& left2 (mask): Sentence 2d]. This masking procedure took place independently of where in the italic region of the breakdown region participants fixated. No masking occurred when the breakdown region was not fixated. Words returned to their original state when the invisible boundary at the fourth character or at the end of the breakdown region was crossed by an eye movement. The UMass eyetracking software initiates display changes within $1 \mathrm{~ms}$. Thus, using a 140$\mathrm{Hz}$ monitor, participants were presented with a masked word within at most $8 \mathrm{~ms}$ after fixating the breakdown region.

(2.) (a) While Mary was mending the clock in the dark hall fri[ghtened] her with its chime.

(b) While Mary was mending the clock in the dark kntt fri[ghtened] her with its chime.

(c) While Mary was mending the clock in the bnch hall fri[ghtened] her with its chime.

(d) While Mary was mending the clock in the bnch kntt fri[ghtened] her with its chime.

Four lists were created, such that each sentence was shown in all four conditions. After each sentence, participants answered a yes-no comprehension question. At the end of the experiment, we asked participants whether they had noticed anything strange during the experiment. Only a small number of people reported a flicker in approximately two or three sentences.

\section{Results}

\section{Errors}

Participants made errors in answering the yes-no comprehension questions on $33.9 \%$ (434) of the experimental trials. Many fewer errors were made in the filler sentences $(4.4 \%)$. The reason for this relatively high error rate was that the experimental trials were designed to cause comprehension difficulties; to answer the questions correctly, the ambiguities of the garden path sentences had to be successfully resolved. For example, the question for Sentence 1 was Could Mary have mended something else than the clock? Because the main goal of the experiment was to induce regressive eye movements, which are an index of processing difficulty, errors on the comprehension questions were to be expected. The error rates differed only slightly between conditions. In condition left1(mask), $35.9 \%$ (115 trials) were answered incorrectly; in condition left2(mask), this number was $31.1 \%$ (101 trials); in condition left1(mask) \& left2(mask), it was 33.1\% (106 trials); and in condition left1(no mask) \& left2(no mask), $32.2 \%$ (103 trials) were answered incorrectly. An ANOVA analysis did not reveal any significant differences (all $F$ s $<2.8$ ).

\section{Analysis of regressions in experimental trials}

With respect to all saccades, participants initiated a regression in $27.1 \%$. In correctly answered trials, $27.4 \%$ of all saccades were regressions, and in incorrect trials, $26.4 \%$ of all saccades were regressions. The breakdown region triggered $11.4 \%$ (1,244 regressions) of all regressions, $10.6 \%$ (779 regressions) in correct and $12.8 \%$ (465 regressions) in incorrect trials.

The perceptual span

In order to test the extents of the perceptual span to the left and right of fixation, regressions and progressions that were initiated from the breakdown region were examined. To investigate the general behavior of regressive eye movements, no distinctions between correct and incorrect trials were made; however, only saccades initiated from at least the fourth character of the breakdown region were included. Thus, $54.8 \%$ (682 regressions) of the regressions from the breakdown region and $68.0 \%(2,707$ progressions $)$ of the progressions from the breakdown region were included in the present analysis. 
Three dependent variables were tested: the length of the first critical saccade, the duration of the last breakdown-region fixation, and the duration of the last breakdown-region gaze. The last breakdown-region fixation was defined as being the last fixation before the eyes left the breakdown region. If the breakdown region was fixated with more than one fixation, these multiple fixations were merged into the last gaze duration.

Three fixed effects were included: masking of left 1 [left1 (mask) vs. left1(no mask)], masking of left2 [left2(mask) vs. left2(no mask)], and saccade direction (SD; regression vs. progression). The data were analyzed by using linear mixed effects (LME) models (Baayen, 2008). LME models are particularly useful for comparing groups that do not comprise the same amount of data. The data of the present experiments were unbalanced because items needed to be divided post-hoc into items in which participants executed a regression or a progression from the breakdown region. In comparison to a repeated measures analysis of variance, an LME model is more robust toward such data.

Figure 1 displays the saccade lengths for both regressions and progressions from the disambiguating region. Effects for left 1 were highly significant $(t=4.55, p<.001$; masked, 11.1 characters; unmasked, 13.2 characters), as were effects of SD ( $t=28.29, p<.001$; regression, 16.2 characters; progression, 8.1 characters). Crucial for our hypothesis, both the left $1 \times \mathrm{SD}$ and left $2 \times \mathrm{SD}$ interactions were significant $(t=2.09, p<.05 ; t=6.98, p<.001)$. Post-hoc analyses showed a significant difference in saccade lengths between masked and unmasked left1 regions when a regression was executed $(t=4.17, p<.001$; masked, 14.2 characters; unmasked, 18.1 characters), but not when a progression was executed $(t=1.85, p>.10$; masked, 8.0 characters; unmasked, 8.2 characters). Although there was a similar trend for the left 2 region, differences between masked and unmasked left 2 regions for both regressions and progressions were not significant $(t=0.93, p>.10 ; t=0.17, p>.10)$.

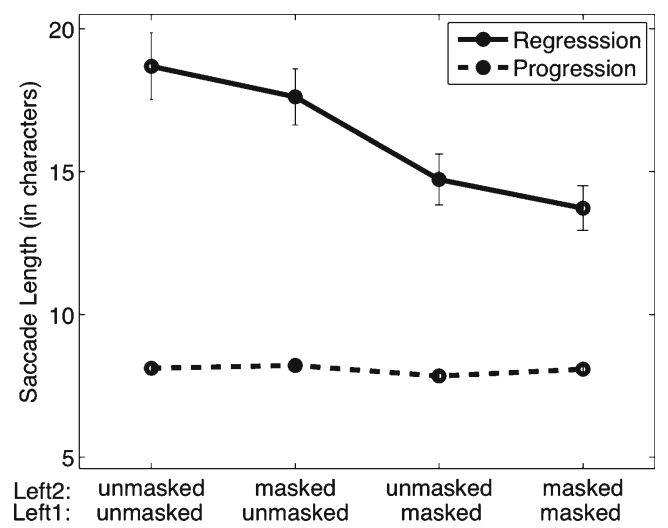

Fig. 1 The length of the first saccade from the breakdown region. The saccade lengths were compared as a function of masking of the first and second words to the left of the breakdown region for regressions and progressions
Similar effects were found for the last fixation duration (Fig. 2) and last gaze duration (Fig. 3) on the breakdown region before either a regression or a progression was launched. Significantly longer fixation durations on the breakdown region were found when the left1 region was masked in comparison with when it was not $(t=2.06, p<.05$; masked, $246 \mathrm{~ms}$; unmasked, $234 \mathrm{~ms}$ ). Importantly, a significant left $1 \times \mathrm{SD}$ interaction was found. Post-hoc analyses showed that the effect of left 1 was marginally significant when the next saccade was a regression ( $t=1.82, p<.07$; masked, $252 \mathrm{~ms}$; unmasked, $231 \mathrm{~ms})$, but not when a progression was going to be launched ( $t=1.21, p>$.10; masked, $240 \mathrm{~ms}$; unmasked, $236 \mathrm{~ms}$ ).

A significant Left $1 \times \mathrm{SD}$ interaction was also found for the duration of the last gaze on the breakdown region before a saccade was launched $(t=3.11, p<.01)$. Post-hoc analyses revealed a significant effect of left1 when a regression was going to be launched $(t=2.90, p<.01$; masked, $339 \mathrm{~ms}$; unmasked, $298 \mathrm{~ms})$, but not for progressions ( $t=0.32, p>.10$; masked, $349 \mathrm{~ms}$; unmasked, $354 \mathrm{~ms})$. Furthermore, effects of SD were also significant $(t=2.03, p<.05)$ : Gaze durations were longer when the next saccade was a progression (351 ms) rather than a regression (319 ms). No other comparisons were significant.

To summarize, with all three dependent variables we found evidence that masking material farther than four characters to the left, and outside of the established perceptual span, influenced reading behavior when the following saccade was a regression. Masking the word to the left of the breakdown region resulted in significantly shorter regressions and longer durations of the last gaze on the breakdown region. Furthermore, marginally longer durations of the last fixations on the breakdown region were found when the word left of the breakdown region was masked in comparison with when it was not masked. In contrast, this manipulation did not change reading behavior when participants executed a progression from the breakdown region.

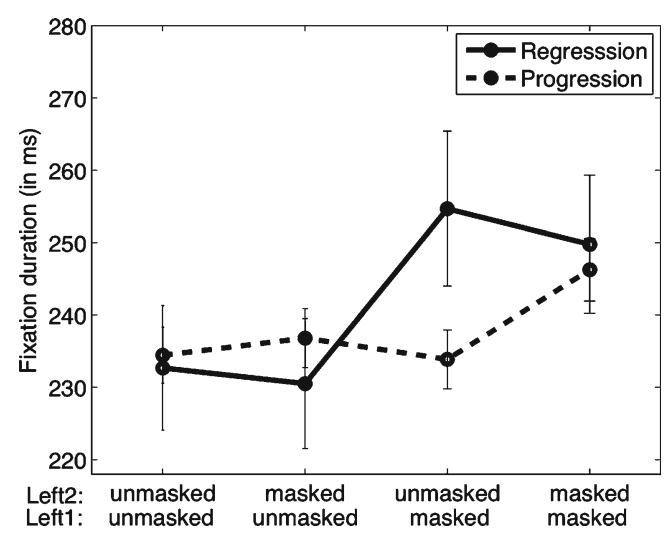

Fig. 2 Mean durations of last fixations before leaving the breakdown region. Fixation durations were compared as a function of masking of the first and second words to the left of the breakdown region for regressions and progressions 


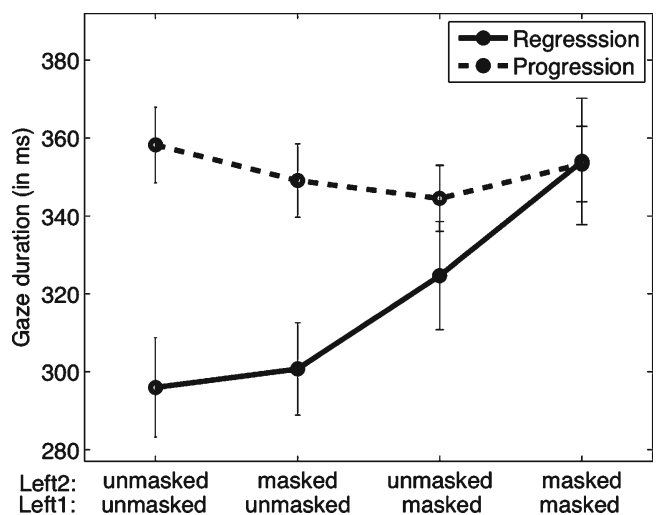

Fig. 3 Mean durations of the last gaze before leaving the breakdown region. Fixation durations were compared as a function of masking of the first and second words to the left of the breakdown region for regressions and progressions

\section{Discussion and conclusion}

The results of this experiment offer clear support for the hypothesis that the perceptual span extends farther than four characters to the left when readers execute regressive eye movements. Masking letters to the left of a current fixation changed reading behavior only when the following saccade was a regression. The manipulation of words to the left did not affect progressive eye movements, which remained at a typical saccade length of eight characters (Rayner, 1998). This result indicates that attention precedes movements of the eyes in whatever direction they are about to move and is supported by similar effects in scene perception (Gersch, Kowler, Schnitzer, \& Dosher, 2009; Henderson et al., 1989).

Although the results of the present study suggest that attention precedes regressive eye movements, it is unclear whether the material to the left is also lexically processed before the eyes move, similar to the parafoveal-on-foveal effects claimed to have been found in forward reading (Kennedy \& Pynte, 2005). The present study did not attempt to answer this question in detail. However, the results suggest at least some processing of the material to the left. Regressions were shorter in the masked condition, and thus were aimed at the masked region. Furthermore, fixation and gaze durations were longer when words were masked, suggesting that readers spent more time reprocessing words to the left. Further evidence that information to the left is lexically processed has also come from an earlier study (Binder, Pollatsek, \& Rayner, 1999). Binder et al. did not investigate regressive eye movements in particular, but they too found that display changes to the left of a fixation changed reading behavior.

In conclusion, the asymmetry of the perceptual span is not statically directed toward the general reading direction of a language. Rather, it depends on the intended direction of a following saccade. Therefore, before people make a regressive eye movement, they shift their attention leftward, which causes the perceptual span in reading to extend farther to the left than the three-to-four-character range it extends during forward reading.

Author note We thank Keith Rayner and one anonymous reviewer for their helpful and constructive comments.

\section{Appendix: Experimental items}

1 The city council argued the position of the radical mayor insinuates a bad immoral outlook.

2 The young ambassador wrote the article which he once used impressed a long lost friend.

3 The hired detective saw the man with a large gun humiliate the scared shop assistant.

4 I suppose he knows the woman wearing that outrageous hat performed with quite crazy stunts.

5 The known criminal confessed his sins which upset many kids disturbed too many kind people.

6 Before the king rides his horse which is very beautiful experiences lots of good care.

7 Wherever Alice enjoys walking her dog which is quite shaggy remembers his usual pet treats.

8 Though Hilda agreed singing the song she had quickly chosen completed in a disappointing way.

9 While Mary was mending the clock in the dark hall frightened her with its chime.

10 As the carpenter builds the table that bends and slopes collapses abruptly in the middle.

11 When the scientist taught the students of biology and chemistry surrounded all the lab equipment.

12 Whilst the janitor polished the floor of the school hall metamorphosized into a beautiful sight.

13 When the cleaners rubbed the paint that ran and dried destroyed the brand new carpet.

14 Because James likes visiting the people who are much older described him with great pleasure.

15 His second wife claims the inheritance which was very large disappeared from his hidden safe.

16 The secretary missed mentioning the error which caused the loss initiated in her own office.

17 This morning, Sam remembered his exams that he was dreading calculated his final year mark.

18 The travel agent confirms the reservation of the young man succeeded over the other bookings.

19 As the journalist wrote the book from the room upstairs increased interest amongst the readers.

20 The speaker happily concluded his lecture which he struggled with relinquished his previous bad reputation.

21 The teacher strongly believed the pupil in the cafeteria abandoned the entire school rules.

22 Since Jay always jogs a mile from school to home definitely seems short to him.

23 The office manager taught the employee that he last chose dominated all the other staff. 
24 The old lecturer wrote the speech that upset most people disrupted all of the politicians.

25 The visitor reads the adverts in the shop window encouraged the people to smoke.

26 Last night, Sally discovered the answer to the difficult problem contradicted her friend's first attempt.

27 The head teacher declared the holiday that he really liked retrieved the old staff morale.

28 After the judge decided the verdict of the long trial captivated the old man's attention.

29 The government memo cautioned the companies that had often lied advertised alcohol to vulnerable youths.

30 The cabinet minister proposed the policy for the new region incorporates great benefits for all.

31 As the woman edited the magazine about fishing in Britain delighted all the reporters.

32 After Mary had drank the water which looked strange evaporated into a purple cloud.

33 Last week, Tom heard the gossip about the new neighbours possesses no truth.

34 The wise historian proves the theory from an Oxford professor reiterates ideas from the past.

35 As the artist paints the picture of the night scene astonishes all the critics greatly.

36 When the ambassador negotiated the treaty about the past events infuriated many of the civilians.

37 Though George continued reading that book about a fictional story extremely bothered him last night.

38 While the motorist parks the lorry that was so noisy whirlwinds along the high street.

39 Whilst zoologists were feeding the tigers from South East India struggled to chew their food.

40 As the cowboys roped the horses that had escaped continued galloping across the ranch.

\section{References}

Baayen, R. H. (2008). Analyzing linguistic data: A practical introduction to statistics using $R$. Cambridge: Cambridge University Press.

Binder, K. S., Pollatsek, A., \& Rayner, K. (1999). Extraction of information to the left of the fixated word in reading. Journal of Experimental Psychology: Human Perception and Performance, $25,1162-1172$.

Frazier, L., \& Rayner, K. (1982). Making and correcting errors during sentence comprehension: Eye movements in the analysis of structurally ambiguous sentences. Cognitive Psychology, 14, 178-210.

Gersch, T. M., Kowler, E., Schnitzer, B. S., \& Dosher, B. A. (2009). Attention during sequences of saccades along marked and memorized paths. Vision Research, 49, 1256-1266. doi:10.1016/ j.visres.2007.10.030

Henderson, J. M., Pollatsek, A., \& Rayner, K. (1989). Covert visual attention and extrafoveal information use during object identification. Perception \& Psychophysics, 45, 196-208. doi:10.3758/ BF03210697

Inhoff, A. W., Pollatsek, A., Posner, M. I., \& Rayner, K. (1989). Covert attention and eye movements during reading. Quarterly Journal of Experimental Psychology, 41A, 63-89.

Kennedy, A., \& Pynte, J. (2005). Parafoveal-on-foveal effects in normal reading. Vision Research, 45, 153-168.

McConkie, G. W., \& Rayner, K. (1975). The span of the effective stimulus during a fixation in reading. Perception \& Psychophysics, 17, 578-586. doi:10.3758/BF03203972

McConkie, G. W., \& Rayner, K. (1976). Asymmetry of the perceptual span in reading. Bulletin of the Psychonomic Society, 8, 365-368.

Meseguer, E., Carreiras, M., \& Clifton, C. (2002). Overt reanalysis strategies and eye movements during the reading of mild garden path sentences. Memory \& Cognition, 30, 551-561.

Morrison, R. E. (1984). Manipulation of stimulus onset delay in reading: Evidence for prallel programming of saccades. Journal of Experimental Psychology: Human Perception and Performance, 10, 667-682.

Pickering, M. J., \& Traxler, M. J. (1998). Plausibility and recovery from garden paths: An eye-tracking study. Journal of Experimental Psychology: Learning, Memory, and Cognition, 24, 940-961.

Pollatsek, A., Bolozky, S., Well, A. D., \& Rayner, K. (1981). Asymmetries in the perceptual span for Israeli readers. Brain and Language, 14, 174-180.

Rayner, K. (1975). The perceptual span and peripheral cues in reading. Cognitive Psychology, 7, 65-81.

Rayner, K. (1979). Eye guidance in reading: fixation locations within words. Perception, 8, 21-30.

Rayner, K. (1998). Eye movements in reading and information processing: 20 years of research. Psychological Bulletin, 124, 372 422. doi:10.1037/0033-2909.124.3.372

Rayner, K., \& Bertera, J. H. (1979). Reading without a fovea. Science, 206, 468-469.

Rayner, K., McConkie, G. W., \& Ehrlich, S. (1978). Eye movements and integrating information across fixations. Journal of Experimental Psychology: Human Perception and Performance, 4, 529 544.

Rayner, K., \& Pollatsek, A. (1989). The psychology of reading. Englwood Cliffs: Prentice Hall.

Rayner, K., Well, A. D., \& Pollatsek, A. (1980). Asymmetry of the effective visual field in reading. Perception \& Psychophysics, 27, 537-544. doi:10.3758/BF03198682

Underwood, N. R., \& McConkie, G. W. (1985). Perceptual span for letter distinctions during reading. Reading Research Quarterly, 20, 153-162.

Vitu, F. (2005). Visual extraction processes and regressive saccades in reading. In G. Underwood (Ed.), Cognitive processes in eye guidance (pp. 1-32). Oxford: Oxford University Press.

Vitu, F., \& McConkie, G. W. (2000). Regressive saccades and word perception in adult reading. In A. Kennedy, R. Radach, D. Heller, \& J. Pynte (Eds.), Reading as a perceptual process (pp. 301-326). Amsterdam: Elsevier. 